$$
\begin{gathered}
\text { CONF-960477--6 } \\
\text { UCRL-JC- }-122798 \\
\text { UCRL-JC-122798 } \\
\text { PREPRINT }
\end{gathered}
$$

\title{
A Unique Data Acquisition System for Electrical Resistance Tomography
}

\author{
W. Daily \\ A. Ramirez \\ K. Zonge
}

This paper was prepared for submittal to the Symposium on the Application of Geophysics to Engineering and Environmental Problems

Keystone, Colorado

April 28-May 1, 1996

January 4, 1996

This is a preprint of a paper intended for publication in a journalor proceedings. Since changes may be made before publication, this preprint is made available with the understanding that it will not be cited or reproduced without the permission of the author. 


\section{DISCLAIMER}

This document was prepared as an account of work sponsored by an agency of the United States Government. Neither the United States Government nor the University of California nor any of their employees, makes any warranty, express or implied, or assumes any legal liability or responsibility for the accuracy, completeness, or usefulness of any information, apparatus, product, or process disclosed, or represents that its use would not infringe privately owned rights. Reference herein to any specific commercial product, process, or service by trade name, trademark, manufacturer, or otherwise, does not necessarily constitute or imply its endorsement, recommendation, or favoring by the United States Government or the University of Califomia. The views and opinions of authors expressed herein do not necessarily state or reflect those of the United States Government or the University of California, and shall not be used for advertising or product endorsement purposes. 


\title{
A Unique Data Acquisition System for Electrical Resistance Tomography
}

\author{
William Daily \\ Abelardo Ramirez \\ Lawrence Livermore National Laboratory \\ Livermore, CA \\ Ken Zonge \\ Zonge Engineering \\ Tucson, AZ
}

\begin{abstract}
Unique capabilities are needed in instrumentation used for acquiring data to do electrical resistance tomography (ERT). A data acquisition system is described which has a good combination of the required capabilities and yet is field rugged and user friendly. The system is a multichannel detector for high data rates, can operate over a wide range of load conditions, will measure both inphase and quadrature resistance at frequencies between $0.0007 \mathrm{~Hz}$ and $8 \mathrm{kjHz}$. The system has been used in both the field and laboratory to collect data with a typical accuracy between 1 and 10\%.
\end{abstract}

\section{BACKGROUND}

Electrical resistance tomography (ERT) is a recently developed method for imaging subsurface electrical resistivity (Tripp et al., 1984; Wexler et al., 1985; Yorkey et al., 1987; LaBrecque and Ward, 1988; Daily and Owen, 1991). The method requires measurement of four electrode resisitivty on many combinations of an array of electrodes placed on the surface and/or in boreholes. Typical electrode arrays range in size from 20 to over 100 electrodes so that tomographic reconstruction from such an array requires a few hundred to thousands of data points. Each datum, the ratio of electrical potential between two electrodes resulting from current flow between two other electrodes, we refer to as a transfer resistance. Another requirement for satisfactory tomographic inversion is that the error in each transfer resistance be small-preferably less than a few percent.

In 1990 when data were first collected for ERT, we used a conventional field resisitivity meter and manually connected to the 
16 electrode array to collect the 104 transfer resistances needed for the computer inversion (Daily et al., 1992). That process required a team of three people working about 3 hours--one person connected leads to the electrodes, one read the resistivity meter and one record data. Even with practice it was a slow, difficult and error-prone task. We quickly realized that an automated data acquisition system was needed. Since none were commercially available we built our own and called it the $\mathrm{R} 2$.

That first automated data acquisition system for ERT used a commercial geophysical resistivity meter to measure the current and voltage and produce the switched DC source current, commercial programmable switches to multiplex the resistivity meter to the electrodes and a computer to control the process and archive the data ( Daily and Ramirez, 1995). This measurement system was capable of measuring about 400 transfer measurements per hour without the element of human error. Under good conditions this system produced data with about $1 \%$ accuracy. In fact, the design worked so well that we built three R2's and still use them today for some field work.

However, as ERT technology developed, it became desirable to add capability and flexibility to the data acquisition system. The first need was for higher data rates. There were several reasons for this. First, 3-dimensional inversions, for which codes were being developed, would require even larger data sets--and field acquisition times. Second, ERT was being used to image dynamic processes like shallow steam floods and it was necessary to "freeze" in time the subsurface changes. The third need was to measure transfer resistance over a range of frequencies since some laboratory data suggested that comparing tomographs at different frequencies may be useful in identifying subsurface contaminants. We also wanted to be able to measure the quadraure transfer resistance (voltage component out of phase with the source current) and use it for tomography. Early in 1994 we began defining the requirements for an instrument which would meet these needs. The result is the subject of this paper which we call the R2D2.

During the past few years several companies building geophysical resistivity instruments have also developed multielectrode data acquisition systems which can be used to collect ERT data. The authors are aware of six such commercial instruments now on the 
market. As far as we know, however, the $\mathrm{R} 2 \mathrm{D} 2$ is unique in capability.

\section{DESCRIPTION OF THE SYSTEM}

R2D2 has the same 4 basic components of the R2: transmitter or current source; receiver which measures the transmitted current as well as the resulting electrode potentials; multiplexer for connecting the electrodes to the transmitter and receiver; a computer for system control and data archival. A block diagram of the system is shown in Figure 1.We now summarize the capabilities of each of these components:

\section{Transmitter}

The current source is powered by a 125 volt, $400 \mathrm{~Hz}$ generator. The output square wave is synchronized with the receiver by an electrically isolated connection or by an internal crystal clock. The output can be regulated to either constant current or constant voltage for a maximum of $3 \mathrm{k}$ watts output.

\section{Receiver}

The heart of the system is a multichannel detector capable of supporting up to 16 modular detectors, each with a10 $\mathrm{M}$ ohm input impedance at DC, a 16-bit A/D converter and a dynamic range from $\pm 0.03 \mu \mathrm{v}$ to $\pm 32 \mathrm{v}$. This large dynamic range is an important attribute when acquiring ERT data since electrode combinations are used in imaging which yield a large variation in received voltage. There is an automatic SP offset adjustment of up to $\pm 2.25 \mathrm{~V}$ in $65 \mu \mathrm{V}$ steps. Each channel has automatic gain setting in binary steps from 1 to 65,536 , but a with manual override the gains may be set externally-a time saving feature when high data rates are needed. Calibration of each channel can be accomplished against either an internal or externally supplied reference voltage.

In practice one channel is used to measure the transmitted current as a voltage drop across a calibrated resistor in the transmitter circuit. This leaves 15 channels for simultaneous potential measurements and is a major reason for the increase in data acquisition rate of the R2D2 over the R2 and other single channel systems. 


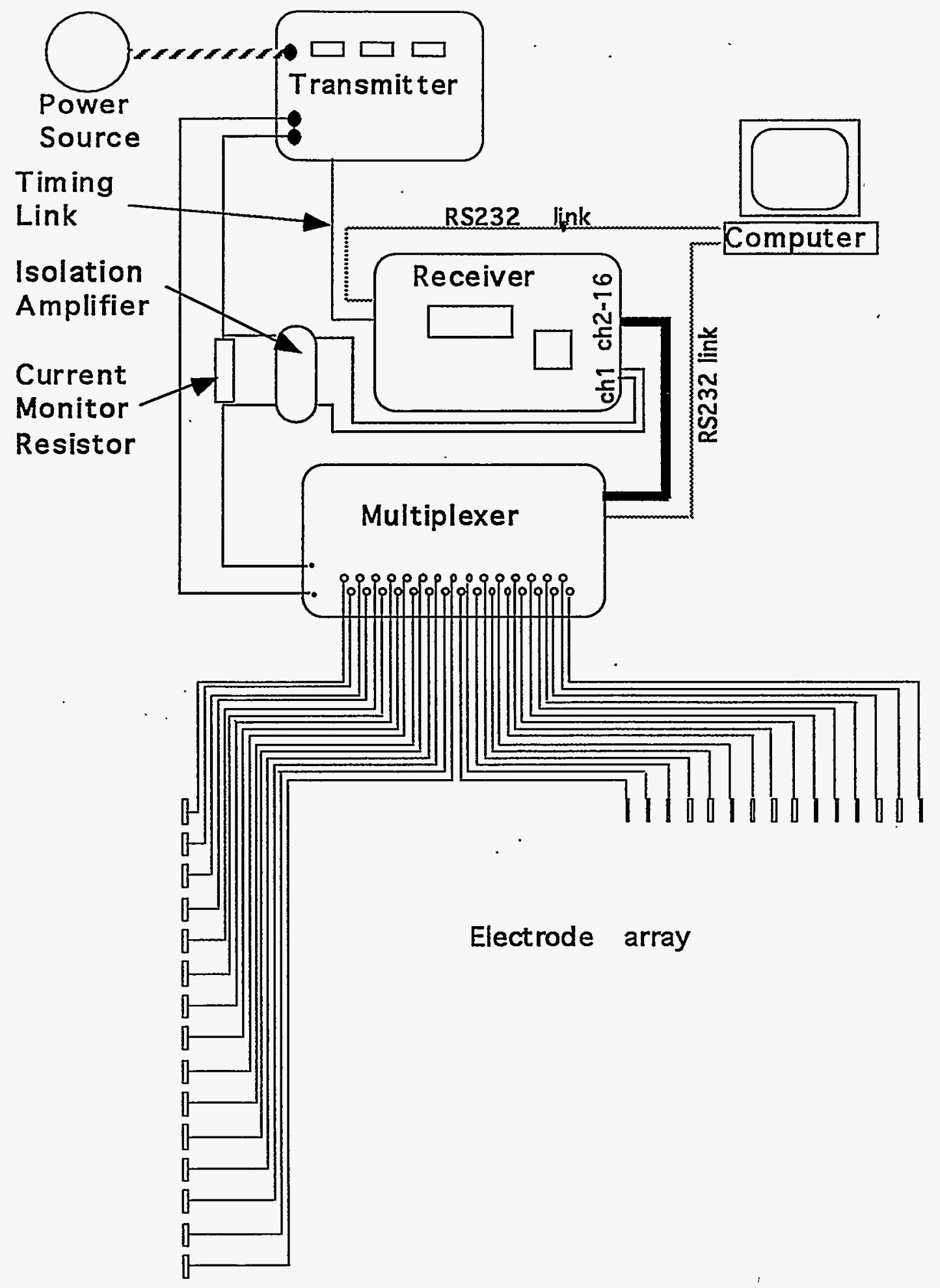

Figure 1. Block diagram of R2D2 system showing the main system components. Additional multiplexers can be daisy-chained to address more than 30 electrodes. The electrode arrays can be on the surface, in boreholes or both. 
The receiver operates in both the frequency domain and time domain. Twenty four frequencies can be selected in binary intervals between $0.0007 \mathrm{~Hz}$ and $8 \mathrm{k} \mathrm{Hz}$ and over this range both the signal magnitude and phase can be measured.

\section{Multiplexer}

Inherent in the switching scheme is the use of "dumb" electrodes. This means that the switching is accomplished at a central point and is not distributed to the individual electrode positions as in many "smart" electrode systems. "Dumb" electrodes require that a separate wire connect the central switching bank (multiplexer) and each electrode. Although this requires more wire and sometimes more field set up time, it makes for disposable electrodes--the only type which can be grouted into boreholes in any significant number. Since our strategy is to position electrodes underground, as close as possible to the imaged region, R2D2 requires "dumb" electrodes with a central multiplexer.

The multiplexier system is modular. Each module can address 30 separate "dumb" electrodes and modules can be daisy-chained together to handle multiples of 30 electrodes. Control of each module is via RS232 protocol such that any electrode can be connected to either high or low side of the current source or the high or low side of any detector. This is a valuable flexibility not found in some commercial systems. Contributing to the overall system data acquisition rate is the switching speed which is $100 \mathrm{~ms}$. The multiplexer switches are rated at 10 amperes at 500 volts.

\section{Computer}

System control is via RS232 protocol from a notebook computer to the receiver and multiplexer. The control software is written in a graphical interface language which is very user friendly.

All of the essential front panel controls on the receiver can be set remotely via the RS232 link: time domain or frequency domain operation, source frequency, staking, analog stage power line filters, amplifier gains. Data is downloaded in real time from the receiver to the computer and displayed so that the operator can easily monitor data acquisition.

The multiplexer control allows for complete freedom in specifying which electrodes are used for current source and which are used for 
potential measurement. This protocol is specified by the user in an ASCII file read by the control program.

\section{SYSTEM PERFORMANCE}

The best evidences of overall performance are the results of field data collected using the R2D2. Such results are presented in this volume by Ramirez et al., (1996)a,b. The following summary of system performance is drawn from experience during those case studies and from laboratory tests

\section{Data acquisition speed}

Data rates are determined by the number of channels which could be continuously used, source frequency and staking requirements. Under normal conditions, at $64 \mathrm{~Hz}$ and staking for $1 \mathrm{sec}$, it was possible to acquire data with about $1 \%$ error (as determined by comparing reciprocal measurements) at a rate of 3077 transfer resistance measurements per hour ( $t r h)$. This data rate could be increased by premeasuring the analog amplifier gains for each measurement and using these gain settings instead of allowing the system to determine them before each measurement. Using prerecorded gains the data rate increased to about 4000 trh. However, prerecorded gains have been used only in laboratory tests and not in field data acquisition.

\section{Data accuracy}

Accuracy is usually defined as "the conformity of an indicated value to an accepted standard value, or true value" (precision, on the other hand, is the "degree of exactness with which a quantity is stated") (Considine, 1983.). As one measure of system accuracy we have performed a simple system calibration against a NIST standard. One channel was used to measure the current through a standard resistor (with a NIST pedigree) while another channel was used to measure the resulting potential drop. Table 1 shows data from calibration of one channel where the measured resistance was accurate to better than $1 \%$. This is a check of the routine field calibration.

Unfortunately, this calibration is only a lower limit of the errors in field data where electrode noise, cable coupling, external noise sources, etc. will combine to increase the errors and decrease accuracy. Maximizing data accuracy on a system where errors may vary from one electrode pair to another, or even change with time, is a difficult problem. Equally important, but just as difficult to obtain, is a reliable measure of accuracy since fitting the model calculations 


\section{Table 1}

\begin{tabular}{lllll}
$\begin{array}{l}\text { NIST } \\
\text { standard resistor, } \\
\text { ohms }\end{array}$ & \multicolumn{5}{c}{ R2D2 measurements, ohms } \\
& first & error & second & error \\
400.03 & 403.42 & $+0.85 \%$ & 402.30 & $+0.57 \%$ \\
100.00 & 100.80 & $+0.80 \%$ & 100.54 & $+0.54 \%$ \\
198.17 & 199.53 & $+0.68 \%$ & 199.14 & $+0.49 \%$ \\
\hline
\end{tabular}

to the measured data to an arbitrarily close tolerance can lead to obviously incorrect inversions (see LaBrecque et al., 1993). However, these are more than academic issues in ERT as data accuracy is an important factor in determining overall image quality.

Several measures are used to estimate data accuracy. The most common is repeatability but our experience has been that this test leads to a serious underestimate of error. We have found that reciprocity is a better estimate of error (for a normal 4 electrode measurement of transfer resistance, the reciprocal is found by interchanging the current source and potential measurement electrodes).

The actual errors estimated using this method are dominated by sources other than the data acquisition electronics: electrode material, formation resistivity, formation electrochemistry, ambient electromagnetic noise, etc. Despite this, it is useful to show here typical errors in field data as an illustration of overall system performance. Figure 2 shows the difference between a normal and reciprocal transfer resistance (our estimate of error) for typical $1 \mathrm{~Hz}$ field data. What is actually plotted is the largest error out of 10 different measurements for each transfer resistance and its reciptrocal so this is therefore an overestimate of error on an individual measurement. Notice that this estimate yields errors typically between about 1 and 10\% and there is a weak correlation with the transfer resistance magnitude. 


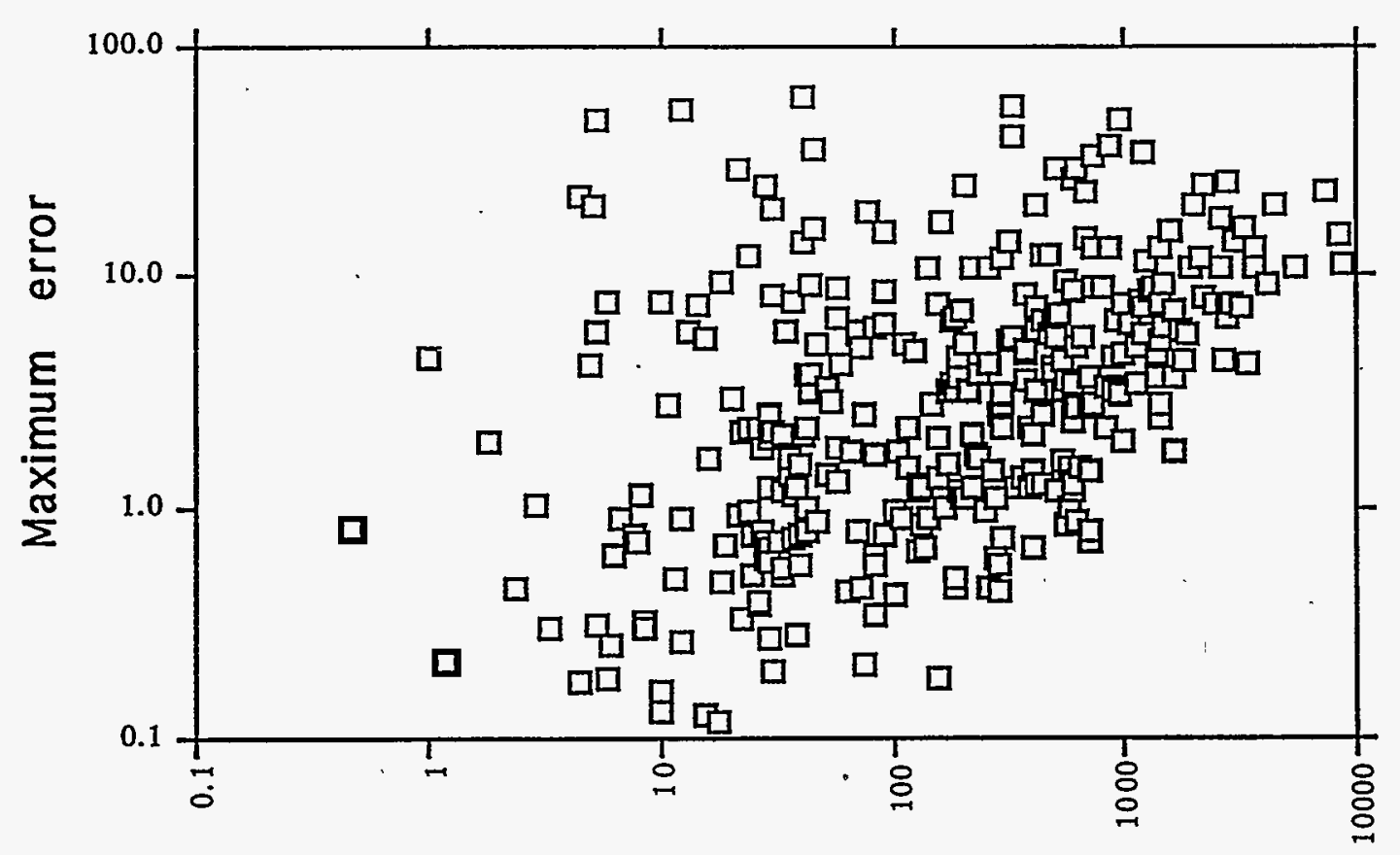

Transfer resistance, milli ohm

Figure 2. Measured error as a function of transfer resitance at a frequency of $1 \mathrm{~Hz}$.

\section{ACKNOWLEDGMENTS}

The authors thank J. Beatty for R2Bं 2 system control software development.

Work performed under the auspices of the U.S. Department of Energy by the Lawrence Livermore National Laboratory under Contract W7405-Eng-48.

\section{REFERENCES}

Considine, D. M., Van Nostrand's Scientific Encyclopedia, Sixth Edition, Van Nostrand Reinhold, Inc., 1983,

Daily, W. and E. Owen, Cross-borehole resistivity tomography, Geophysics, 56, no. 8, 1228-1235, 1991. 
Daily, W. and A. Ramirez, Electrical resistance tomography during insitu trichloroethynene remediation at the Savannah River Site, Applied Geophysics, 33, 239-249, 1995.

Daily, W., A. Ramirez, D. LaBrecque and J. Nitao, Electrical resistivity tomography of vadose water movement, Water Resources Research, 28, no. 5, 1429-1442, 1992.

LaBrecque, D. and S. Ward, Two-dimensional inversion of crossborehole resistivity data using multiple boundaries, 58th Ann. Internat. Mtg, Soc. Expl. Geophys., Expanded Abstracts, 194-197, 1988.

LaBrecque, S. Schima, W. Daily and A. Ramirez, Tracking Air Sparging Using Resistivity Tomography, abstract, EOS American Geophysical Union, Fall Annual Meeting December 6-10, San Francisco, 1993.

Ramirez, A., W. Daily and D. LaBrecque, Complex Electrical Resistivity Tomography of a Subsurface PCE Plume, SAGEEP'96, Keystone, Colorado, 1996a.

Ramirez, A., W. Daily, A. Binley and D. LaBrecque, Detection of Leaks in Underground Storage Tanks using Electrical Resistance Methods, SAGEEP'96, Keystone, Colorado, 1996b.

Tripp, A. C., G. W. Hohmann and C. M. Swift, Two dimensional resistivity inversion, Geophysics, 49, 1708-1717, 1984.

Wexler, A, B. Fry and M. R. Neuman, Impedance-computed tomography algorithm and system, Appl. Optics, 24, 3985-3992, 1985.

Yorkey, T. J., J. G. Webster and W. J. Tompkins, Comparing reconstruction algorithms for electrical impedance tomography, IEEE Trans. Biomed. Eng. BME-34, 843-852, 1987. 


Technical Information Department • Lawrence Livermore National Laboratory University of California • Livermore, California 94551

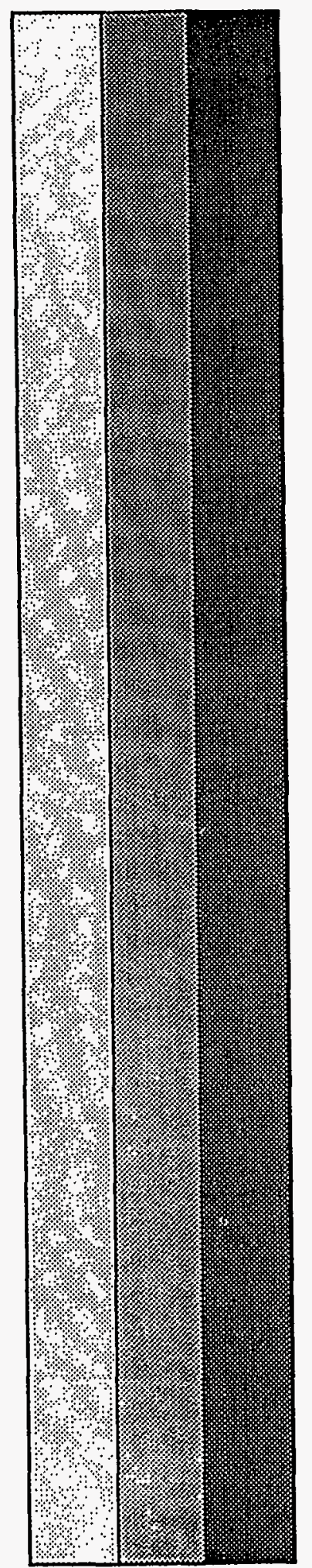

